\title{
Electromagnetic Determination of Carotid Blood Flow in the Anesthetized Rat*
}

\author{
P. COX $\dagger$, H. ARORA $\dagger \dagger$ AND A. KOLIN $\dagger$
}

\begin{abstract}
Summary-The paper describes the design and performance of the smallest iron core electromagnetic flow transducer built so far. The design is miniaturized as to the total volume of the device as well as to the size of the artery it accommodates. The use of this transducer for recording of blood flow in the smallest species of animal employed until now in blood flow research is described. Illustrations of pharmacological observations in anesthetized rats are presented.
\end{abstract}

T HE PROGRESSIVE miniaturization of the electromagnetic flow meter in recent years has enabled blood flow to be measured in the conscious cat in arteries as small as $1.5 \mathrm{~mm}$ in diameter. ${ }^{1}$ There is, however, need for even smaller flow meters which would not only allow the measurement of blood flow to small organs or parts of larger organs but would also permit smaller, inexpensive and more readily available laboratory animals to be used for circulation research. The difficulties of reducing the size of the flow transducer increase very steeply with decreasing artery size. Even the step from a $1.5 \mathrm{~mm}$ to a $1 \mathrm{~mm}$ artery lumen presents considerable problems. The difficulty is due to the great care and precision with which the wiring has to be done to avoid excessive error signals at zero flow and to the need for adequate space for a slot through which the artery is to be inserted and to the smallness of the shutter which seals this slot.

The present paper describes a miniature flow meter for arteries $1 \mathrm{~mm}$ in diameter which has overcome this problem and has been used successfully to record carotid blood flow in anesthetized rats. Fig. 1 shows a photograph of the transducer comparing it to the size of the animal and of an aspirin tablet. Fig. 2(a) shows the magnet core (terminating in the pole pieces $P_{1}, P_{2}$ ) the sleeve $S$ and the wiring ( 85 turns of \#31 gauge Formvar insulated wire) in cross section. Fig. 2(b) shows a perpendicular section of the magnet and of the cylindrical sleeve $S$ (with electrodes $E_{1}$ and $E_{2}$ ) into which a slot [SL of Fig. 2(a)] is cut after potting. Essential details of the wiring and shielding are also shown as well as the slot $S L$ and shutter $S h$ which closes the slot $S L$ when inserted. The EMF induced in the blood as it streams transversely between the poles $P_{1}$ and $P_{2}$ of the opentoroid magnet is picked up by the platinized platinum

* Received August 29, 1963. The work reported in this paper was supported by Grant \#H-3092 of the U. S. Public Health Service, and a Grant from the Office of Naval Research, Washington, D. C.

$\dagger$ Department of Biophysics and Nuclear Medicine, University of California, Los Angeles, Calif.

$\dagger \dagger$ California Institute of Technology, Pasadena, Calif.

${ }^{1}$ A. Kolin and R. T. Kado, "Miniaturization of the electromagnetic blood flow meter," Proc. Natl. Acad. Sci. USA, vol. 45, pp. 1312-1321; August, 1959. electrodes $\left(E_{1}\right.$ and $\left.E_{2}\right)(0.4 \mathrm{~mm}$ in diameter) and conveyed through the twisted leads $L_{1}, L_{2}$ to the gated sinewave amplifying and recording system of the type described in Kolin and Kado. ${ }^{1}$ The magnet is excited by a $400 \mathrm{cps}$ alternating current of 0.6 a rms. Great care is taken in construction of the flow probe to eliminate, or

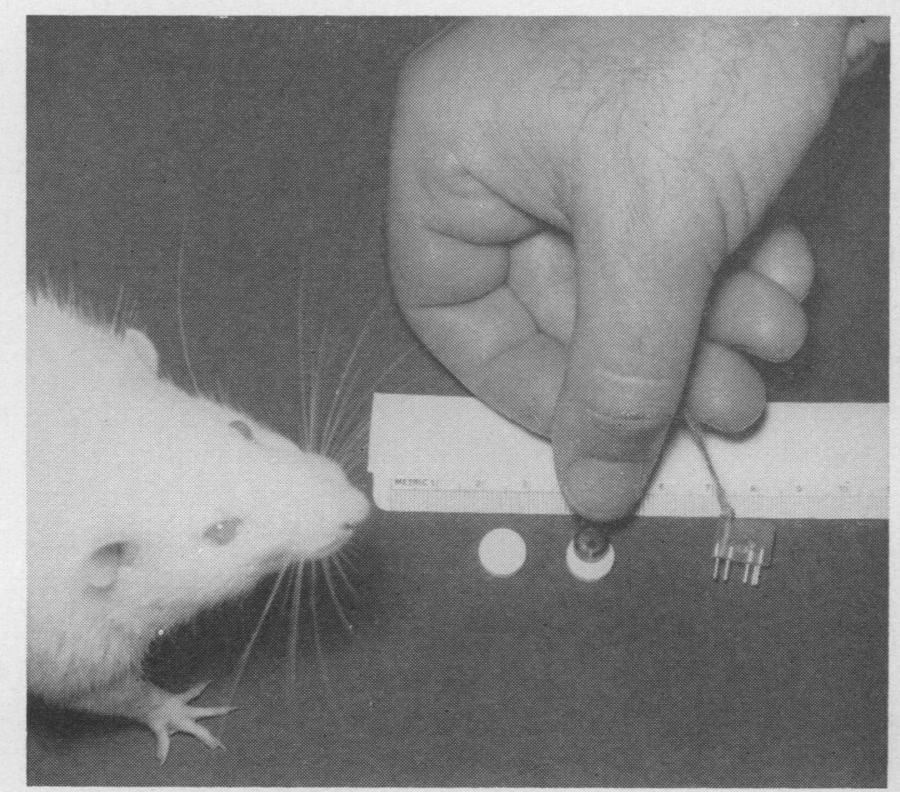

Fig. 1-View of the miniature flow transducer and comparison of its dimensions with those of an aspirin tablet and of the animal.

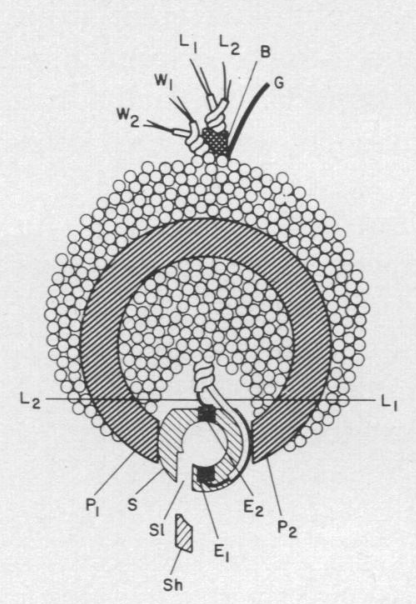

(a)

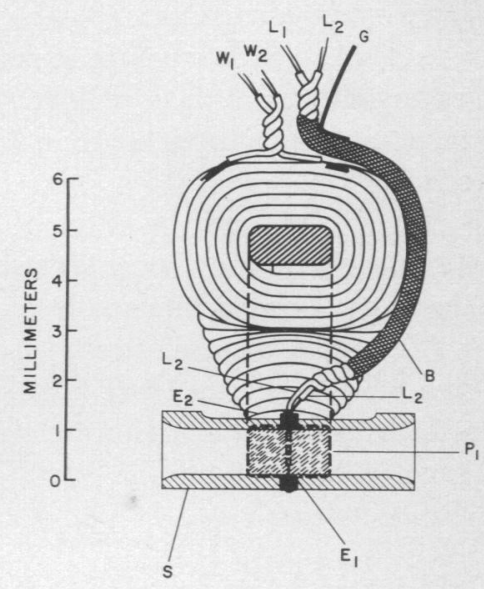

(b)
Fig. 2-(a) Sections of the flow transducer perpendicular to the flow velocity vector and (b) parallel to the flow velocity vector. $W_{1}$, $W_{2}$ : current supply leads to coil energizing the magnet. $L_{1}, L_{2}$ : Electrode lead wires, B: grounded wire braid shielding the electrode leads. G: ground lead. $E_{1}, E_{2}$ : electrodes. $P_{1}, P_{2}$ : magnet poles. $S$ : plastic sleeve. Sl: slot for admission of artery. $S h$ : shutter. 
greatly reduce, the quadrature EMF disturbance as described by Kolin and Wisshaupt. ${ }^{2}$ The platinum electrodes are platinized using the procedure referred to in Kolin and Wisshaupt. ${ }^{2}$ The plastic cylinder ("sleeve" $S$ ) in which the electrodes are mounted is made of epoxy (Hysol \#2038 rod) and the entire assembly of magnet and electrodes is potted in Hysol \#2038 epoxy so that the polyvinyl chloride tubing (not shown in Fig. 2) encasing the wires shown in Fig. 2(a) and (b) is potted over a length of at least $2.5 \mathrm{~mm}$ in the solid epoxy resin. The plug is made by molding of the same plastic material according to a design previously described ${ }^{2}$ in connection with flow meters of a different type. The animal is grounded at a point close to the flow transducer by a separate ground lead. The magnetic field obtained with such flow transducers at a magnet current of 1 a is approximately 290 gauss. Calibration and phase adjustment procedures are described in Kolin and Wisshaupt, ${ }^{2}$ Ryan, ${ }^{3}$ and Glasser. ${ }^{4}$

Fig. 3(a) shows the contour of the blood flow through the left acrotid artery of a 450 -gm rat taken at high speed of the recording paper. The subsequent records of Fig. 3, taken at a slower speed, show respiratory variations in blood flow (measured in the left carotid artery) and blood pressure which was recorded in the right carotid artery by a "Sanborn" physiological pressure transducer. A $20-\mu \mathrm{f}$ condenser in parallel to the recorder input makes it possible to record average values of flow as well as pressure. Fig. 3(b) illustrates the transition from recording of instantaneous values to average values at points $P_{1}$ and $P_{2}$.

Fig. 3(b)-(e) give examples of performance of the miniature flow transducers in observing carotid blood flow in physiological experiments on rats. Blood pressure is recorded at the same time. In Fig. 3(b) the effect of inhalation of amyl nitrite for 7 seconds upon average carotid blood flow and blood pressure is shown. The blood flow drops to 40 per cent of the original value while the blood pressure is reduced to 74 per cent of its value before inhalation. The effect upon the blood flow is thus more pronounced than the effect upon blood pressure.

Fig. 3(c) shows the effect of amyl nitrite inhalation for 10 seconds upon instantaneous values of carotid blood flow and blood pressure.

The next two records illustrate the effect of an intravenously injected drug. Fig. 3(d) shows the effect of $0.125 \mathrm{mg}$ of acetyl choline upon the average values of carotid blood flow and blood pressure. The blood flow drops markedly to 11 per cent of its initial value while

${ }^{2}$ A. Kolin and R. Wisshaupt, "Single-coil coreless electromagnetic blood-flow meters," IEEE Trans. ON Bio-MedicAl ElECTRONics, vol. BME-10, pp. 60-67; April, 1963.

3 D. P. Ryan, "A low cost, portable electromagnetic blood flow meter system, utilizing commercially available devices," IEEE Trans. on Bio-Medical Electronics, vol. BME-10, pp. 57-60; April, 1963.

${ }^{4} \mathrm{O}$. Glasser, "Determination of blood flow by electromagnetic induction," in "Medical Physics," Year Book Medical Publishers, Inc., Chicago, Ill., vol. 3, p. 141; 1960.

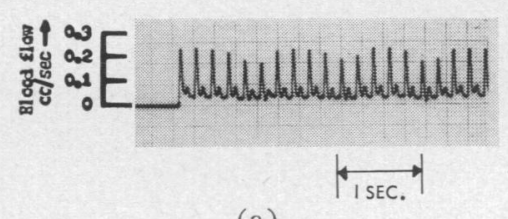

(a)

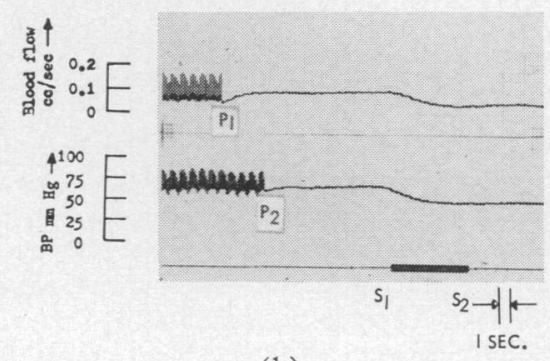

(b)

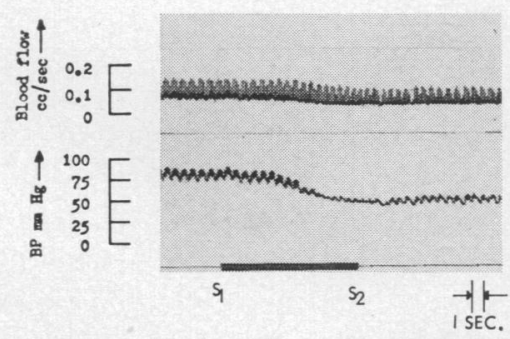

(c)

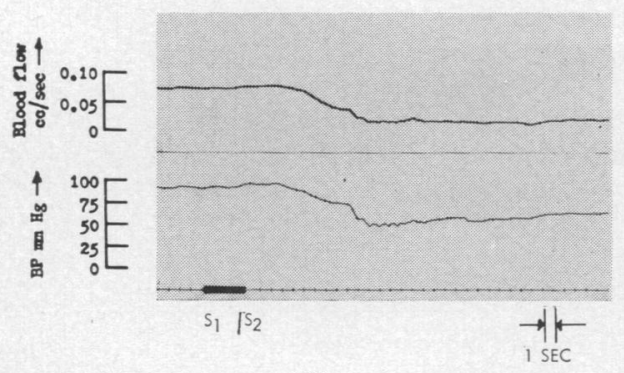

(d)

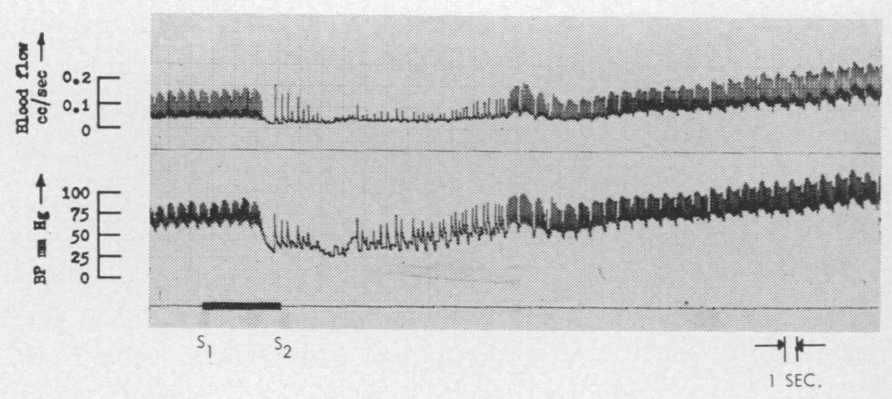

(e)

Fig. 3-Performance of the miniature blood flow transducer in recording blood flow in a rat's left carotid artery $1 \mathrm{~mm}$ in diameter. (a) Tracing of instantaneous blood flow. (b) Upper tracing: blood flow in the left carotid artery. Lower tracing: blood pressure taken in the right carotid artery. The initial portions of the tracings show instantaneous flow and pressure. Points $P_{1}$ and $P_{2}$ mark the transition from observation of phasic values to average values. During the time interval $S_{1}-S_{2}$ the animal inhales amyl nitrate, which leads to pronounced drops in average blood flow and blood pressure. (c) Effect of inhalation (during time interval $S_{1}-S_{2}$ ) of amyl nitrate upon instantaneous carotid blood flow and blood pressure. (d) Effect of $0.125 \mathrm{mg}$ of acetyl choline (injected intravenously between $S_{1}$ and $S_{2}$ ) upon average values of carotid blood flow and blood pressure. (e) Effect of $0.250 \mathrm{mg}$ of acetyl choline (injected intravenously between $S_{1}$ and $S_{2}$ ) upon instantaneous carotid blood flow and blood pressure. This record reveals not only the changes in blood flow and blood pressure but also those in heart rate. 
the blood pressure is decreased to 49 per cent of its value at the outset of the experiment. Again the effect upon blood flow is much more pronounced than upon blood pressure. Fig. 3(e) shows the effect of twice the previous intravenous dose $(0.25 \mathrm{mg})$ upon the instantaneous values of blood flow and pressure revealing the initial effects upon heart rate as well as changes in the pulse volume. The initial diminution in blood flow and blood pressure is followed by a marked subsequent increase.

To our knowledge, the rat is the smallest animal in which blood flow has been recorded by the electromagnetic method and the arterial diameter as well as rate of blood flow are the smallest recorded so far.
The work on futher miniaturization of the flow transducer with the ultimate objective of measuring blood flow in small arterial branches of conscious freely moving animals is in progress and will be reported in greater detail elsewhere.

\section{ACKNowledgments}

The authors gratefully acknowledge the able technical assistance of S. Austin in the performance of the physiological experiments and of D. P. Ryan in connection with the calibrations and operation of the electronic system. Thanks are also due to Dr. Gordon Ross for a critical review of the manuscript.

\title{
Improved Transducer for External Recording of Arterial Pulse Waves*
}

\author{
M. DAVIS $\dagger$, B. GILMORE $\ddagger$, AND E. FREIS $\ddagger$
}

\begin{abstract}
Summary-This paper describes a new instrument with excellent low frequency response for recording arterial pulse wave forms. It uses a fluid filled chamber and a stiff diaphragm to which a set of semiconductor strain gages are cemented.
\end{abstract}

M OST METHODS for recording arterial pulsations externally in man depend upon the application of a sensing device to the skin over a large artery. The periodic pulsation of the arterial segment produces proportionate pressure changes which activate some form of mechanical or electrical transducer.

To determine whether the pulse waves so obtained have diagnostic value, particularly with regard to changes in the elasticity of the arterial walls associated with aging and atherosclerosis, we considered it necessary to develop a method of recording which would not introduce significant instrumental artifacts. The characteristics of an acceptable transducer should include 1) an essentially flat frequency response between approximately one and $20 \mathrm{cps}, 2$ ) sufficient sensitivity to record weak pulsations, 3) compactness and ease of application, and 4) some means for determining the amount of force being applied to the skin overlying the artery.

A recording system was desired which would be responsive to steady state as well as pulsatile pressure fluctuations. This was necessary because the major characteristics of the arterial pulse contour are deter-

* Received April 25, 1963; revised manuscript received August 29, 1963.

$\dagger$ National Bureau of Standards, Washington, D. C.

$\ddagger$ Veterans Administration Hospital, Washington, D. C. mined by its low frequency components.

Preliminary trials with stiff diaphragms applied directly to the skin gave unsatisfactory results. This was due in part to the difficulty in centering directly over the artery which led to uneven flexing of the diaphragm, and in part to irregularities in skin contours overlying certain arteries. To obviate these difficulties, it was decided to use a fluid system between the skin and the diaphragm of the transducer; the fluid was contained in a housing which was rigid except at the surface applied to the skin where it would be sealed by a compliant membrane. Although valuable clinical information was obtained with such capsules connected to commercial strain gage manometers, this combination was rather bulky and did not lend itself to convenient and accurate manipulation. The necessary restriction in the connection between the capsule and the pressure gage also was found to result in phase shifts between input and output signals at frequencies above $12 \mathrm{cps}$.

\section{Construction of Instrument}

Fig. 1 shows a crosssectional view of the instrument designed to fulfill the requirements outlined above. The rigid housing is partitioned into two compartments by a thin metal diaphragm to which two semi-conductor strain gages ${ }^{1}$ are cemented to the upper surface with epoxy. The lower compartment is water-filled and sealed below by a plastic membrane (Saran Wrap ${ }^{\circledR}$ ) and above by a metal diaphragm. The air-filled upper compart-

${ }^{1}$ Micro Systems, Inc. 